\title{
INTEGRABLE SOLUTIONS OF HAMMERSTEIN AND URYSOHN INTEGRAL EQUATIONS
}

\author{
JÓZEF BANAŚ
}

(Received 26 November 1986)

Communicated by R. O. Vyborny

\begin{abstract}
In this paper we prove theorems on the existence of integrable and monotonic solutions of Hammserstein and Urysohn integral equations. The basic tool used in the proof is the fixed point principle for contractions with respect to the so-called measure of weak noncompactness.
\end{abstract}

1980 Mathematics subject classification (Amer. Math. Soc.) (1985 Revision): 47 H 15.

\section{Introduction}

The most frequently investigated integral equation in nonlinear functional analysis are the Hammerstein equation

$$
x(t)=\varphi(t)+\int_{0}^{1} k(t, s) f(s, x(s)) d s, \quad t \in[0,1],
$$

and the Urysohn equation

$$
x(t)=\varphi(t)+\int_{0}^{1} u(t, s, x(s)) d s, \quad t \in[0,1] .
$$

These equations have been studied in several papers and monographs (see for example Krasnosel'skii et al. [10], Zabrejko et al. [14], Appell [1, 2] and references therein).

Existence theorems for equations (1), (2) can be obtained by applying various fixed point principles. For example, the Banach or Schauder principles are most

(C) 1989 Australian Mathematical Society 0263-6115/89 \$A2.00+0.00 
frequently used although other fixed point theorems and other methods are also applicable (see Appell [1, 2], Krasnosel'skii and Zabrejko [11]). Existence theorems for (1) and (2) proved with the help of the above mentioned tools require rather strong hypotheses, and so the result are not entirely satisfactory. A survey of such existence theorems is given in the book of Zabrejko et al. [14] and in the paper of Appell [2].

The goal of this paper is to prove theorems on existence of solutions of the equations (1) and (2) under weaker hypotheses. Roughly speaking we show that an assumption on the monotonocity of the functions generating the Hammerstein and Urysohn operators (with respect to some variables) allows us to show that these equations have solutions in the space $L^{1}$. The basic tool which will be used is the fixed point theorem due to Emmanuele [7].

The technique used in this paper was introduced in the paper of the author [4] where some functional equations were investigated.

\section{Notation, auxiliary facts and preliminary results}

Let $L^{1}(a, b)$ denote the space of Lebesgue integrable functions on the interval $[a, b]$, with the standard norm

$$
\|x\|=\int_{a}^{b}|x(t)| d t .
$$

For simplicity, we shall consider the space $L^{1}=L^{1}(0,1)$.

This section is mainly devoted to recall some auxiliary results which will be needed further on.

Denote by $S$ the set of all functions which are measurable on $[0,1]$. When we furnish it with the metric

$$
\rho(x, y)=\inf [a+\operatorname{meas}\{s:|x(s)-y(s)| \geq a\}: a>0]
$$

then $S$ becomes a complete metric space. Moreover, it is well known that the convergence in measure coincides with convergence generated by the metric $\rho$ (see Dunford and Schwartz [6]). The convergence in measure of a sequence $\left\{x_{n}\right\} \subset L^{1}$ does not imply the weak convergence of this sequence and conversely. Nevertheless, we have the following result.

LEMMA 1 (Krasnosel'ski et al. [10]). If a sequence $\left\{x_{n}\right\} \subset L^{1}$ converges weakly to $x \in L^{1}$ and is compact in measure then it converges in measure to $x$.

Apart from this we recall that a sequence $\left\{x_{n}\right\} \subset L^{1}$ is convergent strongly (that is, in the norm of $L^{1}$ ) to $x$ if and only if it converges in measure to $x$ and is weakly compact. 
Now, let $E$ denote an arbitrary Banach space and let $X$ be a nonempty and bounded subset of $E$. Moreover, denote by $K_{r}$ the closed ball in $E$ centered at $\Theta$ and with radius $r$. Recall, that the notion of the measure of weak noncompactness $\beta$ was defined by De Blasi [5] in the following way

$$
\begin{aligned}
& \beta(X)=\inf \{r>0: \text { there exists a weakly compact subset } \\
& \left.\qquad Y \text { of } E \text { such that } X \subset Y+K_{r}\right\} .
\end{aligned}
$$

The function $\beta(X)$ possesses several useful properties which may be found in De Blasi's paper [5]. This paper contains also the comparison between the measure $\beta(X)$ and classical measures of noncompactness. A convenient and handy formula for the function $\beta(X)$ in the space $L^{1}$ was given recently by Appell and De Pascale [3]

$$
\beta(X)=\lim _{\varepsilon \rightarrow 0}\left\{\sup _{x \in X}\left\{\sup \left\{\int_{D}|x(t)| d t: D \subset[0,1], \text { meas } D \leq \varepsilon\right\}\right\}\right\} .
$$

The usefulness of the measure of weak noncompactness $\beta$ was pointed out by Emmanuele [7], who proved the following fixed point theorem.

THEOREM 1. Let $Q$ be a nonempty, closed, convex, bounded subset of $E$, and let $T: Q \rightarrow Q$ be a weakly continuous transformation (that is, mapping weakly convergent sequences into itself) having the propety that there is a constant $k \in[0,1)$ such that

$$
\beta(T X) \leq k \beta(X)
$$

for any nonempty subset $X$ of $Q$. Then $T$ has at least one fixed point in the set $Q$.

In the sequel we shall need some criteria for compactness in measure. The complete description of compactness in measure was given by Fréchet [6] but the following sufficient condition will be more convenient for our purposes.

LEMMA 2 (Krasnosel'skii et al. [10]). Let $X$ be a bounded subset of $L^{1}$ and suppose that there is as a family of measurable subsets $\left\{\Omega_{c}\right\}_{0 \leq c \leq 1}$ of the interval $[0,1]$ such that meas $\Omega_{c}=c$. If for any $c \in[0,1]$ and for any $x \in X$

$$
x\left(t_{1}\right) \leq x\left(t_{2}\right) \quad\left(t_{1} \in \Omega_{c}, t_{2} \notin \Omega_{c}\right)
$$

then the set $X$ is compact in measure.

Next we pay our attention to the so-called superposition operator $(F x)(t)=$ $f(x, t(t))$. Assume that $f(x, t)=f:[0,1] \times R \rightarrow R$ satisfies Carathéodory conditions, that is, it is measurable in $t$ for any $x$ and continuous in $x$ for almost all $t$. Then we have the following theorem due to Krasnosel'skii [9]. 
THEOREM 2. The superposition operator $F$ is a continuous map of the space $L^{1}$ into itself if and only if $|f(t, x)| \leq a(t)+b|x|$ for all $t \in[0,1]$ and $x \in R$, where $a(t)$ is a function in $L^{1}$ and $b$ is a nonnegative constant.

The last part of this section will be devoted to preliminary results.

Let $r>0$ be a fixed number. Denote by $Q_{r}$ the subset of the ball $K_{r}$ in the space $L^{1}$ consisting of all functions which are a.e. nondecreasing on the interval $[0,1]$. The set $Q_{r}$ is nonempty, bounded, convex and closed in $L^{1}$ (see Banas [4]). Moreover, according to Lemma 2 this set is compact in measure. Indeed, to show this it suffices to put $\Omega_{c}=[0, c]-P$ for any $c \in[0,1]$, where $P$ denotes a suitable subset of $[0,1]$ with meas $P=0$.

LEMMA 3. Let $\left\{x_{n}\right\} \subset Q_{r}$ be weakly convergent to $x \in L^{1}$. Then $\left\{x_{n}\right\}$ converges strongly to $x$ and $x \in Q_{r}$.

PROOF. Taking into account the fact that $Q_{r}$ is comapct in measure, in virtue of Lemma 1 we infer that $\left\{x_{n}\right\}$ converges in measure to $x$. Hence, keeping in mind the weak compactness of a sequence $\left\{x_{n}\right\}$ we obtain that $\left\{x_{n}\right\}$ converges strongly to $x$. In fact, $x \in Q_{r}$ because $Q_{r}$ is closed. Thus the proof is complete.

COROLLARY. A subset $X$ of $Q_{r}$ is weakly compact if and only if it is compact.

Moreover, we have the following trivial but useful assertion.

LEMMA 4. Any continuous operator $S: Q_{r} \rightarrow L^{1}$ is weakly continuous.

REMARK. In the case when $S: L^{1} \rightarrow L^{1}\left(\right.$ or $S: K_{r} \rightarrow L^{1}$ ) the above lemma is not true according to the result of Shragin [12].

\section{Main results}

Int his section we prove existence theorems for the equations (1) and (2).

First we will consider the Hammerstein equation (1). For convenience the Hammerstein operator

$$
(H x)(t)=\int_{0}^{1} k(t, s) f(s,, x(s)) d s
$$

will be written as product $H=K F$ of the superposition operator

$$
(F x)(t)=f(t, x(t))
$$


and the linear integral operator

$$
(K x)(t)=\int_{0}^{1} k(t, s) x(s) d s
$$

Then the equation (1) has the form $x=B x$ where

$$
B x=\varphi+H x=\varphi+K F x .
$$

Let us assume the following

(i) the function $\varphi \in L^{1}$ is a.e. nondecreasing on the interval $[0,1]$,

(ii) $f:[0,1] \times R \rightarrow R_{+}$satisfies Carathéodory conditions and there exist a function $a \in L^{1}$ and a constant $b \geq 0$ such that

$$
|f(t, x)| \leq a(t)+b|x|
$$

for all $t \in[0,1]$ and $x \in R$,

(iii) $k:[0,1] \times[0,1] \rightarrow R$ is measurable with respect to both variables and such that the integral operator $K$ (defined above) maps $L^{1}$ into itself.

Let us recall that the above assumption implies (Krasnosel'skii et al. [10]) that the operator $K$ maps the space $L^{1}$ continuously into itself. In what follows by $\|K\|$ we denote the norm of the operator $K$.

Further, we assume that

(iv) the function $t \rightarrow k(t, x)$ is a.e. nondecreasing on the interval $[0,1]$ for almost all fixed $s \in[0,1]$,

(v) $b\|K\|<1$.

Then we have the following result.

THEOREM 3. Under the above assumptions the equation (1) has at least one solution $x \in L^{1}$ which is a.e. nondecreasing on the interval $[0,1]$.

PROOF. Let us take an arbitrary $x \in L^{1}$. Then, according to the assumptions (i), (ii), (iii) and Theorem 2 we have that $B x \in L^{1}$ where $B$ is the operator defined in (3). Moreover, we get

$$
\begin{aligned}
\|B\| & \leq\|\varphi\|+\|K F x\| \leq\|\varphi\|+\|K\|\|F x\| \\
& =\|\varphi\|+\|K\| \int_{0}^{1}|f(s, x(s))| d s \\
& \leq\|\varphi\|+\|K\| \int_{0}^{1}[a(s)+b \mid x(s) \|] d s \\
& \leq\|\varphi\|+\|K\|\|a\|+b\|K\|\|x\| .
\end{aligned}
$$

From the above estimate we conclude that the operator $B$ maps the ball $K_{r}$ into itself, where $r=(\|\varphi\|+\|K\|\|a\|) /(1-b\|K\|)$.

Now let $Q_{r}$ denote the subset of $K_{r}$ consisting of all functions which are a.e. nondecreasing on $[0,1]$ (see the last part of Section 2). From the assumptions 
(iv) and (ii) we deduce that $B$ maps $Q_{r}$ to itself. Apart from that, in view of Lemma 4 , we infer that $B$ is weakly continuous on the set $Q_{r}$.

In what follows we show that the operator $B$ is a contraction with respect to the measure of weak noncompactness $\beta$. To do this let us fix $\varepsilon>0$ and $X \subset Q_{r}$. Further, take $x \in X$ and $D \subset[0,1]$ such that meas $D \leq \varepsilon$. Then by our assumptions we obtain

$$
\begin{aligned}
\int_{D}|(B x)(t)| d t & \leq \int_{D}|\varphi(t)| d t+\int_{D}\left|\int_{0}^{1} k(t, s) f(s, x(s)) d s\right| d t \\
& =\|\varphi\|_{L^{1}(D)}+\|K F x\|_{L^{1}(D)} .
\end{aligned}
$$

Now, taking into account that the operator $K$ maps the space $L^{1}(D)$ into itself and is continuous, we get

$$
\begin{aligned}
\int_{D}|(B x)(t)| d t & \leq\|\varphi\|_{L^{1}(D)}+\|K\|_{D}\|F x\|_{L^{1}(D)} \\
& \leq\|\varphi\|_{L^{1}(D)}+\|K\|_{D} \int_{D^{\prime}}[a(s)+b \mid x(s) \| d s \\
& \leq\|\varphi\|_{L^{1}(D)}+\|K\|\|a\|_{L^{1}(D)}+b\|K\|\|x\|_{L^{1}(D)} \\
& =\int_{D}|\varphi(t)| d t+\|K\| \int_{D} a(t) d t+b\|K\| \int_{D}|x(t)| d t
\end{aligned}
$$

where the symbol $\|K\|_{D}$ stands for the norm of the operator $K: L^{1}(D) \rightarrow L^{1}(D)$.

Further, using the obvious fact

$$
\lim _{\varepsilon \rightarrow 0}\left\{\sup \left[\int_{D}|\varphi(t)| d t+\|K\| \int_{D} a(t) d t: D \subset[0,1], \text { meas } D \leq \varepsilon\right]\right\}=0
$$

we have $\beta(B X) \leq b\|K\| \beta(X)$. This inequality together with the assumption (v) and the properties of the set $Q_{r}$ quoted in Section 2 allow us to apply Theorem 1. This completes the proof.

REMARK 1. It is very difficult to find necessary and sufficient conditions for the function $k(t, s)$ guaranteeing that the linear integral operator $K$ maps $L^{1}$ into itself. In some special cases this problem was solved by Juberg [8] and Stuart [13] (see also Zabrejko et al. [14], where some particular cases were also discussed).

REMARK 2. Up to now existence theorems for the equation (1) have been proved under more restrictive assumptions than (ii) and (iii). Namely, it was assumed that $F$ is the so-called "improving" operator while $K$ was supposed to be a regular operator. Conditions of this type were first formulated by Zabrejko and Pustyl'nik [15] (see also $[10,14,2]$ ). 
Throughout the remainder of this section we shall discuss the solvability of the Urysohn equation (2). Denote by $G$ the operator associated with the right-hand side of this equation and by $U$ the Urysohn operator

$$
(U x)(t)=\int_{0}^{1} u(t, s, x(s)) d s .
$$

We assume the following hypotheses:

(i) $u(t, s, x)=u:[0,1] \times[0,1] \times R \rightarrow R$ satisfies the Carathéodory conditions, that is, $u$ is measurable with respect to $(t, s)$ for any $x \in R$ and continuous in $x$ for almost all $(t, s)$,

(ii) the Urysohn operator $U$ maps $L^{1}$ into $L^{1}$ and is continuous,

(iii) the function $t \rightarrow u(t, s, x)$ is a.e. nondecreasing on the interval $[0,1]$ for almost all $s \in[0,1]$ and for each $x \in R$,

(iv) $|u(t, s, x)| \leq k_{1}(t, s)(a(t)+b|x|)$ for $(t, s) \in[0,1]^{2}$ and $x \in R$, where $a \in L^{1}, 0 \leq b=$ const. and a function $k_{1}:[0,1]^{2} \rightarrow R_{+}$is measurable and such that the linear integral operator $K_{1}$ generated by $k_{1}$ is a continuous mapping of $L^{1}$ into itself for which $b\left\|K_{1}\right\|<1$,

(v) $\varphi \in L^{1}$ and is a.e. nondecreasing on the interval $[0,1]$.

Then we can formulate the following theorem.

THEOREM 4. Let the assumptions (i)-(v) be satisfied. Then the equation (2) has at least one solution $x$ such that $x \in L^{1}$ and is a.e. nondecreasing on $[0,1]$.

The proof may be carried over in the same fashion as the proof of Theorem 3 and is therefore omitted. We give only a few comments concerning the assumptions (ii) and (iv).

First of all let us remark that instead of (ii) we can assume that $|k(t, s, x)| \leq$ $p(t, s, x)$, where $p:[0,1] \times[0,1] \times R \rightarrow R$ satisfies Carathéodory conditions and is such that the integral operator

$$
(P x)(t)=\int_{0}^{1} p(t, s, x(s)) d s
$$

maps the space $L^{1}$ into $L^{1}$ and is continuous. Then the continuity of the Urysohn operator $U$ follows from the known majorant principle given in the book in Krasnosel'skii et al. [10], for example. Particularly, as the function $p$ we can take a function determining a Hammerstein operator [14]. We mention also that some conditions guaranteeing the continuity of the operator $U$ may be found in [10].

Finally let us observe that in our considerations we can replace nondecreasing functions by nonincreasing ones. 


\section{Acknowledgements}

The author expresses his gratitude to the referee for some valuable remarks and improvements of the presentation.

\section{References}

[1] J. Appell, 'Implicit functions, nonlinear integral equations, and the measure of noncompactness of the superposition operator', J. Math. Anal. Appl. 83 (1981), 251-263.

[2] J. Appell, 'On the solvability of nonlinear noncompact problems in function spaces with applications to integral and differential equations', Boll. Un. Mat. Ital. B (6) 1 (1982), 1161-1177.

[3] J. Appell and E. De Pascale, 'Su alcuni parametri connesi con la misura di non compattezza di Hausdorff in spazi di functioni misurabili', Boll. Un. Mat. Ital. B (6) 3 (1984), $497-515$.

[4] J. Banas, 'On the superposition operator and integrable solutions of some functional equations', Nonlinear Anal. TMA (preprint).

[5] F. S. De Blasi, 'On a property of the unit sphere in a Banach space', Bull. Math. Soc. Sci. Math. R. S. Roumanie (N.S.) 21 (1977), 259-262.

[6] N. Dunford and J. Schwartz, Linear operators (Interscience, Leyden, 1963).

[7] G. Emmanuele, 'Measure of weak noncompactness and fixed point theorems', Bull. Math. Soc. Sci. Math. R. S. Roumanie (N.S.) 25 (1981), 353-358.

[8] R. K. Juberg, 'The measure of noncompactness in $L^{p}$ for a class of integral operators', Indiana Univ. Math. J. 23 (1974), 925-936.

[9] M. A. Krasnosel'skii, 'On the continuity of the operator $F u(x)=f(x, u(x))$ ', Dokl. Akad. Nauk SSSR 77 (1951), 185-188 (in Russian).

[10] M. A. Krasnosel'skii, P. P. Zabrejko, J. I. Pustyl'nik and P. J. Sobolevskii, Integral operators in spaces of summable functions (Nauka, Moscow, 1966) [English translation: Noordhoff, Leyden, 1976].

[11] M. A. Krasnosel'skii, and P. P. Zabrejko, Geometric methods of nonlinear analysis (Moscow 1975; in Russian).

[12] V. I. Shragin, 'On the weak continuity of the Nemytskii operator', Uchen. Zap. Moskov. O.P.I. 57 (1957), 73-79 (in Russian).

[13] C. A. Stuart, 'The measure of noncompactness of some linear integral operators', Proc. Roy. Soc. Edinburgh 71 (1973), 167-179.

[14] P. P. Zabrejko, A. I. Koshelev, M. A. Krasnosel'skii, S. G. Mikhlin, L. S. Rakovshchik and V. J. Stecenko, Integral equations (Nauka, Moscow, 1968; in Russian).

[15] P. P. Zabrejko and J. I. Pustyl'nik, 'On the continuity and complete continuity of nonlinear integral operators in $L^{p}$ spaces', Uspehi Mat. Nauk 19 (1964), 204-205 (in Russian).

Department of Mathematics

I. Lukasiewicz Technical University

35-084 Rzeszów, Poznańska 2

Poland 